\title{
Endobronchial valves: a bridge to definitive surgical management in COVID-19 recurrent pneumothorax
}

\author{
Joanne Szewczyk ${ }^{1}$, Brian Cody Adkinson ${ }^{2}$, Sisir Akkineni ${ }^{2}$, Dao M. Nguyen ${ }^{1}$, Sixto A. Arias ${ }^{3}$, \\ Nestor R. Villamizar ${ }^{1}$ \\ ${ }^{1}$ Section of Thoracic Surgery, Division of Cardiothoracic Surgery, The DeWitt Daughtry Department of Surgery, University of Miami Miller School \\ of Medicine, Miami, FL, USA; ${ }^{2}$ Division of Pulmonary Medicine, University of Miami Miller School of Medicine, Miami, FL, USA; ${ }^{3}$ Section of \\ Interventional Pulmonology, Division of Pulmonary Medicine, University of Miami Miller School of Medicine, Miami, FL, USA \\ Correspondence to: Nestor R Villamizar, MD. University of Miami, Section of Thoracic Surgery, 1611 NW 12 ${ }^{\text {th }}$ Avenue, Miami, FL 33136 , USA. \\ Email: nvillamizar@med.miami.edu.
}

Submitted Nov 07, 2020. Accepted for publication Nov 18, 2020.

doi: 10.21037/jtd-20-3248

View this article at: http://dx.doi.org/10.21037/jtd-20-3248

Pneumothorax associated with COVID-19 can occur with or without pre-existing lung disease or mechanical ventilation, may completely resolve, or recur requiring definitive surgical intervention (1). In patients with active severe acute respiratory syndrome coronavirus 2 (SARS-CoV-2) infection undergoing surgery, pulmonary complications occur in half of the patients and there is an associated increased in mortality, as high as $20 \%$ (2). Therefore, careful consideration should be given to these patients requiring a surgical intervention, with preference for alternative, conservative modality if feasible.

In 2018, the FDA approved two valves for bronchoscopic lung volume reduction-the Zephyr endobronchial valve (PulmonX) and the Spiration valve system (Olympus) (3). Endobronchial valves (EBV) can be used for occlusion of a bronchopleural fistula causing persistent pneumothorax and prolonged air leak as a treatment for late complications after lung resection (4). Here, we describe the first use of Zephyr endobronchial valves in COVID-19-associated pneumonia to remedy persistent air leak in the critical care setting as a bridge to definitive surgical treatment.

A 69-year-old male with chronic obstructive pulmonary disease and severe bullous emphysema presented to our institution with shortness of breath for four days. On admission, he was found to have COVID-19 pneumonia and a large, spontaneous, left pneumothorax requiring tube thoracostomy. After one week, he developed worsening acute hypoxic respiratory failure requiring intensive care unit admission. Computed tomography angiography
(CTA) of the chest showed persistent pneumothorax despite adequate tube thoracostomy and a new large, saddle pulmonary embolism requiring catheter-directed thrombolysis (Figure 1). A second tube thoracostomy was performed with persistent air leak and lack of resolution of pneumothorax. He continued to develop worsening acute hypoxic and hypercapnic respiratory failure requiring intubation and mechanical ventilation.

Given his declining clinical status, a multidisciplinary discussion was held to determine the best approach for management of his persistent air leak. Due to his current frail status, high morbidity and mortality associated with an intervention during an active COVID-19 infection, he was deemed a poor surgical candidate, thus endobronchial management was pursued.

Seventeen days from initial tube thoracostomy, and eight days after intubation, both chest tubes exhibited persistent grade 5 air leak with $150 \mathrm{~mL}$ air leak per tidal volume. Flexible bronchoscopy was performed and a balloon catheter was deployed into each subsegmental bronchus while monitoring the intensity of air leak. The leak was markedly attenuated by complete obstruction of the left upper lobe. Zephyr EBVs were separately deployed to the left upper lobe apicoposterior segment, anterior segment, and lingula. Afterward, the air leak diminished to a grade 1 with only $30 \mathrm{~mL}$ air leak per tidal volume. The patient was extubated two days later. Follow-up chest CT showed complete resolution of pneumothorax and subsequently the chest tubes were removed. The patient was discharged 22 days 


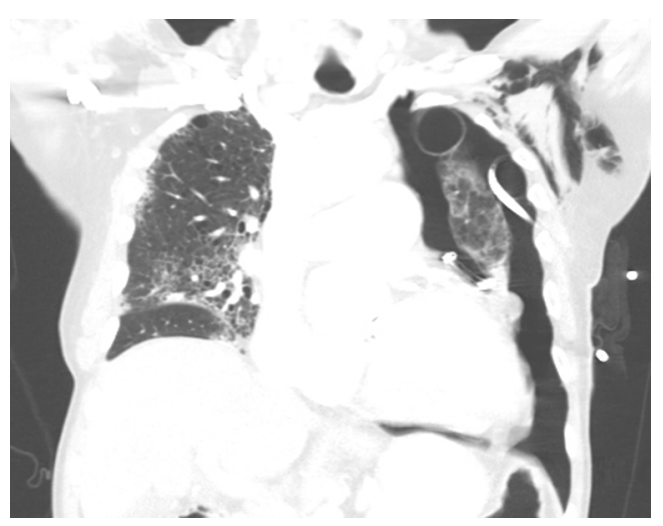

Figure 1 Computed tomography angiography (CTA) of the chest showed persistent pneumothorax despite adequate tube thoracostomy.

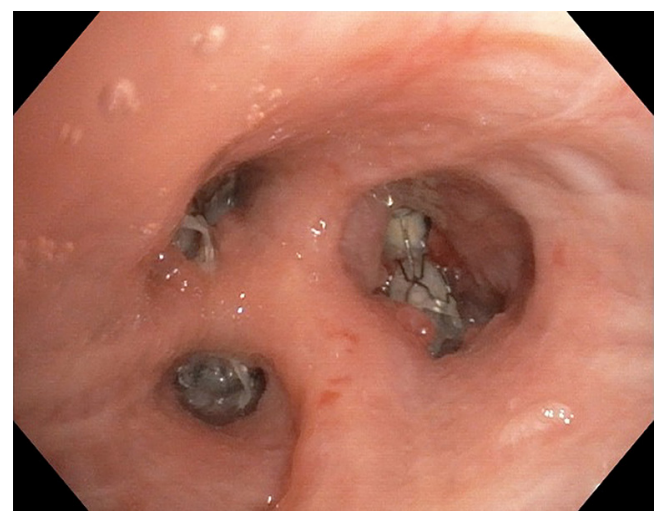

Figure 2 Bronchoscopy view of left upper lobe with the three endobronchial valves (EBVs) visible. The anterior segment EBV has migrated.

after the EBV placement.

Over one month later, during outpatient pulmonary follow up, CT chest demonstrated a recurrent left pneumothorax requiring readmission and pigtail pleural catheter placement. At this time, patient was without evidence of an active COVID-19 infection. Bronchoscopy demonstrated adequate positioning of the apicoposterior and lingular valves with mild rotation of the anterior segment valve (Figure 2). The three EBVs were removed. Since his pneumothorax was related to his underlying bullous emphysema and unlikely to resolve without intervention, definitive surgery was decided upon. The patient underwent left video assisted thoracoscopic bullectomy, decortication, and talc pleurodesis and was discharged without any air leak.
With the advent of the COVID-19 pandemic, we have seen an unprecedented number of patients with pneumothorax and persistent air leak after tube thoracostomy. According to a recently published large, multicenter case series, roughly $1 \%$ of patients admitted with COVID-19, and up to $15 \%$ of mechanically vented patients, develop pneumothorax, often in association with acute hypoxic respiratory failure and a protracted clinical course. ${ }^{1}$ The American College of Chest Physicians recommends surgical evaluation if an air leak does not resolve spontaneously after four days of tube thoracostomy (5). A persistent air leak while mechanically ventilated can lead to ventilation/perfusion mismatch, difficulty maintaining PEEP, and inability to heal due to positive pressure (6). In addition, there is also a theoretical risk that persistent air leaks in a COVID-19 patient can cause increased aerosolization of the virus during changing of chest tube drainage systems, placing health care providers at risk of infection (7). Conservative treatment with chest drain and observation is usually effective for the treatment of COVID-19 pneumothorax complicated by a persistent air leak, but invasive techniques are needed when conservative treatment fails (6).

Unfortunately, the increased risk of mortality for patients undergoing thoracic surgery with a concomitant active COVID-19 infection makes the timing of a definitive operation a challenging decision highlighting the need for an alternative, less invasive approach $(8,9)$. Endobronchial valves $(\mathrm{EBV})$ historically have been used in the management of lung volume reduction surgery but has gained acceptance in the management of patients with spontaneous pneumothorax and persistent air leaks, especially in those who are poor surgical candidates $(6,10)$. Our case demonstrates the utility of EBVs in the critical care setting, especially during the COVID-19 pandemic, when patients are too high risk for acute surgical treatment of pulmonary pathologies. To the best to our knowledge, this is the $1^{\text {st }}$ case report describing the use of EBV as a bridge therapy in the management of pneumothorax during active COVID-19 disease.

With the increasing finding of pneumothorax due to COVID-19, we highlight the utility of EBVs as a viable strategy to reduce the morbidity of COVID-19-associated bronchopleural fistulas in the critical care setting and offers a strategic bridge to definitive surgical treatment at a more opportune time. Appropriate patient selection for EBV placement is essential and a multidisciplinary discussion needs to be held between intensive care unit, 
interventional pulmonology and thoracic surgery for optimal results. Though the endobronchial valve does not always offer definitive repair as in our case, it may still offer a temporizing solution until the patient has sufficiently recovered from the initial insult to allow for future definitive intervention.

\section{Acknowledgments}

Funding: None.

\section{Footnote}

Provenance and Peer Review: This article was a free submission to the journal. The article did not undergo external peer review.

Conflicts of Interest: All authors have completed the ICMJE uniform disclosure form (available at http://dx.doi. org/10.21037/jtd-20-3248). The authors have no conflicts of interest to declare.

Ethical Statement: The authors are accountable for all aspects of the work in ensuring that questions related to the accuracy or integrity of any part of the work are appropriately investigated and resolved.

Open Access Statement: This is an Open Access article distributed in accordance with the Creative Commons Attribution-NonCommercial-NoDerivs 4.0 International License (CC BY-NC-ND 4.0), which permits the noncommercial replication and distribution of the article with the strict proviso that no changes or edits are made and the original work is properly cited (including links to both the formal publication through the relevant DOI and the license). See: https://creativecommons.org/licenses/by-nc-nd/4.0/.

Cite this article as: Szewczyk J, Adkinson BC, Akkineni S, Nguyen DM, Arias SA, Villamizar NR. Endobronchial valves: a bridge to definitive surgical management in COVID-19 recurrent pneumothorax. J Thorac Dis 2021;13(1):411-413. doi: 10.21037/ jtd-20-3248

\section{References}

1. Martinelli AW, Ingle T, Newman J, et al. COVID-19 and Pneumothorax: A Multicentre Retrospective Case Series. Eur Respir J 2020;56:2002697.

2. COVID Surg Collaborative. Mortality and pulmonary complications in patients undergoing surgery with perioperative SARS-CoV-2 infection: an international cohort study. Lancet 2020;396:27-38.

3. FDA Center for Devices and Radiological Health. (19 April 2019). Zephyr ${ }^{\circledR}$ Endobronchial Valve System P180002. Retrieved 6 October 2020. Available online: https://www.fda.gov/medical-devices/recently-approveddevices/zephyrr-endobronchial-valve- system-p180002

4. Snell GI, Holsworth L, Fowler S, et al. Occlusion of a broncho-cutaneous fistula with endobronchial one-way valves. Ann Thorac Surg 2005;80:1930-2.

5. Baumann MH, Strange C, Heffner JE, et al. Management of Spontaneous Pneumothorax An American College of Chest Physicians Delphi Consensus Statement consensus conference 590 Consensus Conference. Chest 2001;119:590-602.

6. Dugan KC, Laxmanan B, Murgu S, et al. Management of Persistent Air Leaks. Chest 2017;152:417-23.

7. Sugimoto H, Kohama T. Chest tube with air leaks is a potential "super spreader" of COVID-19. Am J Infect Control 2020;48:969.

8. Peng S, Huang L, Zhao B, et al. Clinical course of coronavirus disease 2019 in 11 patients after thoracic surgery and challenges in diagnosis. J Thorac Cardiovasc Surg 2020;160:585-92.

9. Cai Y, Hao Z, Gao Y, et al. Coronavirus Disease 2019 in the Perioperative Period of Lung Resection: A Brief Report From a Single Thoracic Surgery Department in Wuhan, People's Republic of China. J Thorac Oncol 2020;15:1065-72.

10. Qi F, Tian Q, Chen L, et al. Use of endobronchial valve insertion to treat relapsing pneumothorax: a case report and literature review. Clin Respir J 2017;11:411-8. 